University of Nebraska - Lincoln

DigitalCommons@University of Nebraska - Lincoln

Faculty Publications from Nebraska Center for

Materials and Nanoscience, Nebraska Center Materials and Nanoscience

for (NCMN)

$5-15-2003$

\title{
Multiscale modeling of hysteretic phenomena in magnets „(invited)
}

Vladimir P. Antropov

Ames Laboratory, US Department of Energy, antropov@ameslab.gov

Kirill D. Belashchenko

University of Nebraska-Lincoln, belashchenko@unl.edu

Follow this and additional works at: https://digitalcommons.unl.edu/cmrafacpub

Part of the Nanoscience and Nanotechnology Commons

Antropov, Vladimir P. and Belashchenko, Kirill D., "Multiscale modeling of hysteretic phenomena in magnets „(invited)" (2003). Faculty Publications from Nebraska Center for Materials and Nanoscience. 24.

https://digitalcommons.unl.edu/cmrafacpub/24

This Article is brought to you for free and open access by the Materials and Nanoscience, Nebraska Center for (NCMN) at DigitalCommons@University of Nebraska - Lincoln. It has been accepted for inclusion in Faculty Publications from Nebraska Center for Materials and Nanoscience by an authorized administrator of DigitalCommons@University of Nebraska - Lincoln. 


\title{
Multiscale modeling of hysteretic phenomena in magnets (invited)
}

\author{
V. P. Antropov a) and K. D. Belashchenko \\ Ames Laboratory, Ames, Iowa 50011
}

(Presented on 12 November 2002)

\begin{abstract}
Methodology of multiscale modeling of hysteretic phenomena in magnets is discussed. A practical combination of first-principles, micromagnetic, and microstructural calculations is constructed which allows one to study the hysteretic phenomena in hard magnets. Advantages and shortcomings of this approach are discussed. Multiscale nature of coercivity in CoPt type magnets is elucidated. Two sources of coercivity in polytwinned CoPt type magnets developing at different length scales, domain wall pinning at antiphase boundaries and splitting at twin boundaries, are illustrated for a realistic microstructure. (C) 2003 American Institute of Physics. [DOI: 10.1063/1.1556096]
\end{abstract}

\section{INTRODUCTION}

Many physical processes involve a wide range of spacial and temporal length scales. Sometimes the physics of such processes may be drastically simplified due to some kind of scaling whereupon the problem becomes essentially singlescale. This is especially characteristic for systems close to a critical point. The properties of such systems may be described by a small set of macroscopic parameters containing all necessary information about microscopic interactions.

The problem of coercivity presents an example of a different sort. Several length scales of different nature participate nontrivially in the physics of magnetization reversal. Exchange interaction and magnetocrystalline anisotropy (MCA) are acting on the microscopic scale, while the longrange dipole-dipole interaction essentially has a length scale of the magnetic flux closure distance which depends dynamically on the magnetic structure. Other length scales pertain to the microstructure of a magnet which has a crucial effect on the properties of hard magnets. Notably, a diversity of spatial and temporal scales produces properties which would otherwise be unattainable. ${ }^{1}$

The notion of multiscale modeling (MSM) stems from the necessity to treat inherently multiscale problems of this type. The most direct approach is to include several degrees of freedom on an equal footing and to perform large-scale computer simulations ("brute force" solution) in the frame of a single scale technique. Unfortunately, traditional methods face great difficulties when applied to multiscale problems. For instance, $a b$ initio techniques which are very fruitful for systems with the effective size of 1 to $2 \mathrm{~nm}$, when applied to direct domain wall $(5-100 \mathrm{~nm})$ modeling, ${ }^{2}$ practically do not produce new physical insight while having unsatisfactory numerical accuracy and requiring a huge amount of computational time. "Smarter" single scale techniques, which may employ different spatial or temporal resolution, such as wavelets, multigrids, or different coarse graining techniques have been announced on both $a b$ initio and model levels, ${ }^{3,4}$ but practical applications are very limited due to enormous computational efforts. In both cases the

\footnotetext{
${ }^{a)}$ Electronic mail: antropov@ameslab.gov
}

current research is limited to model systems and cannot be applied for practical simulations of realistic materials.

Therefore it seems that a more fruitful path is to proceed by analogy with the scaling theories by seeking a simplified description replacing the effect of certain degrees of freedom with the corresponding effective dynamics. Two types of techniques may be used on this path. One is to use some effective equations which are suitable only for one particular scale whereas the influence of others is taken into account via some phenomenological material constants. The other technique involves bringing several theoretical tools with different regions of applicability under one roof. As we will show below, currently it is the most practical approach for the description of hysteretic phenomena in real materials. It also seems to be quite general, and we believe that it may be used for both spacial and, to a lesser extent, temporal scales. Before we proceed with the detailed description of methods, let us analyze the existing scales in applied magnetism.

Let us first discuss the different temporal scales which may be associated with the dynamics of typical magnets. Starting from the slowest mechanisms, the first temporal scale is that of magnetic domains in the applied magnetic field. This scale corresponds to the propagation of domain walls and increase in the size of domains which have a net magnetization parallel to the applied field. The key physical quantities involved are exchange and dipole-dipole interactions and MCA. The second scale concerns the magnetization reversal of a single domain. It occurs when the energy brought by the field overcomes the anisotropy energy barrier. The time scale associated with this mechanism $\left(10^{-10}-10^{-8} \mathrm{~s}\right)$ depends on extrinsic factors. The third important scale is that of the damping of the magnetization dynamics. On the microscopic level it corresponds to dissipation of energy stored in the magnetic system into lattice vibrations. The corresponding processes have the characteristic time scale of $10^{-10}-10^{-11} \mathrm{~s}$. Several models describing magnetization dynamics on this scale were used for years. In Bloch, Landau-Lifshitz, or Gilbert dynamics this damping is taken into account via phenomenological relaxation terms. Such dynamic equations have a generic nature, and the presence of a damping term is the standard way of imitating the effect of microscopic degrees of freedom which collectively 
produce the equilibrium magnetization. So, even the simple model of magnetization dynamics provided by the LandauLifshitz equation reflects the same philosophy of scale separation.

The shortest times correspond to quantum fluctuations of the magnetization. This is a region of fast and ultrafast spin dynamics. This time scale is irrelevant to the dynamics of domain walls and effectively can be taken into account by employing the adiabatic approximation. It corresponds, for instance, to local changes of the magnetization which result from the broad energy spectrum, $W \simeq 3-5 \mathrm{eV}$. A time scale of the order $1 / W\left(10^{-15} \mathrm{~s}\right)$ and larger is expected.

So far not so many interesting temporal multiscale problems have been considered. In general, temporal MSM is rather limited and in the majority of cases the "brute force" approach ("let us run the simulation longer") prevails. So, below we will limit our consideration of magnetization reversal (MR) to the static case and concentrate only on problems with multiple length scales. Unfortunately, the relevant spatial scales in the description of applied magnetic phenomena are too numerous and diverse to be described here. Below we emphasize the most generic features using hysteretic phenomena in a particular group of hard magnets as an example.

It is a well established experimental fact that the hysteretic properties of a magnetic material are very sensitive both to its composition and microstructure. The studies of purely magnetic phenomena on the nanosize scale (domain wall structure, shape of domains, and so on) can usually be addressed with appropriate micromagnetic techniques (e.g., the finite element method ${ }^{5,6}$ ). Quite extended regions (typically with the size of up to $\sim 100 \delta$ where $\delta$ is the domain wall width) may be studied with these techniques, but such important ingredients of the problem as the atomic-scale properties of defects and the microstructure are usually addressed only phenomenologically. The microstructure plays an especially important role in the properties of hard magnets because their high coercivity always develops in certain characteristic nanoscale microstructures containing a high density of such defects as grain boundaries, twins, interphase and antiphase boundaries (APB), etc. Already here it is evident that the theoretical description of the effect of microstructure on the hysteresis loop of a hard magnet presents a rather complex task due to the inherently multiscale nature of the problem and the presence of several entirely different interactions. Interaction of domain walls with many important defects is determined by variations of the microscopic interaction parameters within the regions of atomistic size $(\sim 1 \mathrm{~nm})$. The domain wall width $(5-10 \mathrm{~nm}$ in most hard magnets) is another length scale, while the microstructure itself has one or more additional length scales (typically within the 10-200 nm range). Each of these length scales is physically important, and all of them must be linked together in order to describe the magnetic properties consistently. Therefore the inclusion of magnetic interactions on different length scales (from atomistic to submicron) and elastic interactions (responsible for a given microstructure) seems to be important for the consistent description of the hysteretic phenomena.
Reliable description of the properties of defects may currently be obtained only in first-principles calculations. The simplest way to obtain the required physical insight is to convert the results of such studies into the form of a suitable effective Hamiltonian making the coupling between the geometry and energetics of domain walls more transparent. Moreover, in setting up a technique compatible with microstructural and micromagnetic simulations, we are forced to use the model approach.

The key parameters produced by electronic structure calculations are interatomic exchange coupling, spin stiffness tensor, and MCA. Let us discuss how these parameters are defined in the band structure theory.

The intersite exchange parameters have been calculated many times using very different methods within the local density approximation (LDA). To date, all numerical calculations for real materials used an expression where the effective exchange is pairwise and proportional to the bare magnetic susceptibility $J_{i j}=I^{2} m_{i} \chi_{i j} m_{j}$, where $I$ is the intraatomic exchange. Such an expression represents a long wave expansion of a more general expression $J_{i j}=m_{i}[\chi]_{i j}^{-1} m_{j}$ which has never been used in the computational materials science. For instance, the most practical multiple scattering expression

$$
J_{i j}=\frac{1}{4 \pi} \int^{\varepsilon_{F}} d \varepsilon \operatorname{Im}_{\operatorname{Tr}_{L}}\left(T^{\uparrow}-T^{\downarrow}\right)_{i i}\left[T^{\dagger} T^{\downarrow}\right]_{i j}^{-1}\left(T^{\uparrow}-T^{\downarrow}\right)_{j j},
$$

where $T_{i i}^{\uparrow}$ is a $T$ matrix of scattering, has not been explored. Moreover, the exact expression for the adiabatic spin wave spectra $^{7} \omega(\mathbf{q})=m\left[\chi_{0}^{-1}(0)-\chi_{0}^{-1}(\mathbf{q})\right]$, where $\chi_{0}(\mathbf{q})$ is "bare" magnetic susceptibility, rigorously takes into account the itinerant character of spin excitations and naturally leads to the larger dispersion compared to the one produced by localized (atomistic) Heisenberg model $\omega_{\mathrm{loc}}(\mathbf{q})$ $=m I^{2}\left[\chi_{0}(\mathbf{q})-\chi_{0}(0)\right]$. Correspondingly, the largest error of localized approximation appears for the nearest atoms exchange, affecting the critical temperature of magnetic phase transition. Fortunately, in the long-wave approach, in spite of the approximate character of each $J_{i j}$, the spin wave stiffness $D_{\alpha \beta}$ can be studied rigorously. However, it should be done with some caution, because the real space expression $D_{\alpha \beta} \sim \sum J_{i j} R_{i}^{\alpha} R_{j}^{\beta}$ is essentially pairwise and is not taking into account possible multisite exchange contributions. The nonpairwise interactions are important in itinerant and localized magnets as temperature increases and they must be included in any calculations to be realistic.

In cubic magnets $D_{\alpha \beta}$, being a second-rank tensor, reduces to a scalar which is proportional to the micromagnetic exchange constant $A$. However, in noncubic materials including all known hard magnets this is not the case. In particular, in hexagonal and tetragonal magnets this tensor has two nonequal (in-plane, $D_{\perp}$ and out-of-plane, $D_{\|}$) components, so that $\omega(\mathbf{q}) \simeq D_{\perp}\left(q_{x}^{2}+q_{y}^{2}\right)+D_{\|} q_{z}^{2}$ at small $q$. This anisotropy of spin stiffness may have an important effect on the hysteretic properties by generating a preference in the domain wall orientation. Although in structures characterized by a sufficiently large length scale this preference is masked by dominating magnetostatic interactions, peak coercivity in hard 
magnets is achieved in nanoscale microstructures where effects of spin stiffness anisotropy should be quite pronounced. First-principles calculations may provide valuable information about spin stiffness anisotropy which is rather hard to obtain from experiment.

Whereas the qualitative considerations above have been around for a long time, to our knowledge, no theoretical methods combining the atomistic, microstructural, and $\mathrm{mi}-$ cromagnetic parts of the multiscale coercivity problem in real systems have been suggested. Below we show how one can combine the first-principles, micromagnetic, and microstructural calculations and apply them to study the rich physics of hysteretic phenomena in hard magnets of CoPt type and demonstrate that coercivity has a natural multiscale character and must be studied using an approach including all the relevant scales.

\section{CHOICE OF A PROTOTYPE SYSTEM}

The majority of widely used permanent magnets belong to two groups of rare earth-transition metal (RETM) alloys ( $\mathrm{Nd}-\mathrm{Fe}-\mathrm{B}$ type and $\mathrm{Sm}-\mathrm{Co}$ type). High coercivity achieved in these magnets is due to their high magnetocrystalline anisotropy and certain characteristic microstructures. The most important defects in $\mathrm{Nd}-\mathrm{Fe}-\mathrm{B}$ type magnets are the grain boundaries, sometimes with segregated intergranular phases. Modern precipitation-hardened $\mathrm{Sm}_{2} \mathrm{Co}_{17}-\mathrm{SmCo}_{5}$ magnets develop a peculiar cellular microstructure ${ }^{1}$ which is crystallographically coherent and dominated by interphase boundaries, lamellar inclusions, and twins. Among the main factors governing the coercivity of different RETM magnets are exchange decoupling at grain boundaries and domain wall pinning at interfaces. A major problem in the treatment of granular $\mathrm{Nd}-\mathrm{Fe}-\mathrm{B}$ magnets is the lack of information about the microscopic structure of the grain boundaries. In many cases the magnetic parameters associated with the grain boundaries are unknown, and guesswork is inevitably involved in the simulations. Sm-Co magnets seem to be more tractable in this respect due to crystallographic coherency of their microstructure, but reliable first-principles calculations of the defect properties at present are impossible due to the strongly correlated character of samarium $f$-electrons (the same problem pertains to $\mathrm{Nd}-\mathrm{Fe}-\mathrm{B}$ magnets as well). In addition, magnetic properties of $\mathrm{Sm}-\mathrm{Co}$ magnets depend strongly on doping; the most widely used variety contains additions of $\mathrm{Fe}, \mathrm{Cu}$, and $\mathrm{Zr}$. These additions strongly modify the magnetic properties of the phases present in the microstructure. While consistent multiscale studies of $\mathrm{Sm}-\mathrm{Co}$ magnets seem to be possible, they are certainly quite complex and involve many degrees of freedom. It is desirable to work out the methodology using a more simple material with no technical problems (like $f$ electrons).

Intermetallic hard magnets $\mathrm{CoPt}, \mathrm{FePt}$, and FePd developing high coercivities in the tetragonal $L 1_{0}$ phase are quite suitable for this task. All the accumulated knowledge about this magnet family suggests that it can serve as a prototype for the problem at hand. First of all, they are single-phase, and their main microstructural features are well established experimentally on the nanoscale level $(10-100 \mathrm{~nm})$. The mi- crostructure is dominated by crystallographically coherent twins and APBs. ${ }^{8,9}$ This circumstance provides two major advantages: the microstructure may be consistently simulated theoretically (see Sec. III), while the microscopic properties of defects are tractable for modern first-principles methods based on the density functional theory (DFT). Indeed, the description of electronic structure of alloys of $3 d$ atoms with $\mathrm{Pd}$ and $\mathrm{Pt}$ is sufficiently reliable, and the range of perturbation near an APB (1-5 nm) may also be covered. Such parameters as magnetic anisotropy and effective exchange coupling are also routinely computed. With this methodical background, identification of the links between the microstructure and magnetic properties of bulk CoPt-type magnets is of interest both for the understanding of their own physics, and for the general theory of hysteretic phenomena.

\section{MICROSTRUCTURE: POLYTWINNING AND ANTIPHASE BOUNDARIES}

Typical thermal processing for CoPt type alloys involves high-temperature annealing in the disordered $A 1$ (fcc) phase area of the phase diagram followed by a rapid quench and aging at a lower temperature in the $L 1_{0}$ phase area. Microstructural evolution during aging includes two stages, ${ }^{8}$ the "tweed" stage and the twinning stage. The "tweed contrast" corresponds to a uniform pattern formed by small (1-10 nm) ordered domains with a dominant $\{101\}$ orientation of interfaces and essentially random distribution of the $c$-axis directions (pointing along one of the three cubic axes of the parent fcc lattice). When the growing ordered domains reach a certain characteristic size $(10-20 \mathrm{~nm})$, a new pattern develops, with the formation of large "polytwinned" stacks containing ordered "bands" ("c-domains") with two alternating directions of the $c$ axis making $\pi / 2$ angles with each other. The interfaces between the $c$ domains within a polytwinned stack (twin boundaries) are all parallel to each other and lie in one of the $\{101\}$ planes.

Physically, polytwinning is explained by the fact that such structures eliminate the volume-dependent part of the elastic energy stemming from the coexistence of ordered domains with different $c$-axis directions. ${ }^{10}$ Recently a microscopic model for the description of elastic interactions in alloys undergoing $L 1_{0}$ type ordering was used ${ }^{11}$ in detailed studies of various aspects of microstructural evolution. In such alloys the elastic interaction is effectively nonpairwise, but it retains the standard long-wavelength elastic singularity. ${ }^{10,11}$ Therefore, the elastic interaction, as the dipole-dipole (magnetostatic) interaction, formally has an infinite range. However, due to the fact that the elastic energy of a nonpolytwinned array of ordered domains grows faster with the domain size compared to the contribution from the interface tension, there is a characteristic size $l_{0}$ at which the elastic interaction begins to affect the microstructural evolution. ${ }^{11}$ This size (usually about $10 \mathrm{~nm}$ ) corresponds to the average size of domains on the tweed stage; the thickness of twins on the polytwinned stage cannot be less than $l_{0}$.

Another generic feature of the polytwinned stage is a large density of APBs within the twinned bands. As we show below in Sec. IV, the APBs act as pinning centers for domain walls due to a local suppression of the magnetocrystalline 
anisotropy (MCA). Clearly, the efficiency of pinning depends on the pattern formed by APBs. So far, little is known about the dependence of this pattern on the alloy composition and thermal processing. For example, in Fig. 5 of Ref. 12 the APBs in a CoPt sample appear to be rectilinear, while the available TEM images ${ }^{8}$ for FePd alloys reveal quite dense patterns of curved APBs, albeit with some tendency to a preferred alignment. The former case is readily reproduced in microscopic simulations, ${ }^{11}$ while the prerequisites for the latter pattern are unclear. It is quite obvious that pinning efficiency should strongly depend on the prevailing APB pattern, and each case should be studied separately. One way to understand the combined effect of macrodomain wall splitting and pinning in various microstructures is to take characteristic microstructures obtained in microscopic simulations and add the magnetic degrees of freedom using the method described in Ref. 17. We will illustrate this approach in Sec. VII for the easily reproduced case of rectilinear APBs in $\mathrm{CoPt}$.

\section{PINNING AT ANTIPHASE BOUNDARIES}

If APB is represented micromagnetically as a slab of thickness $w \ll \delta\left[\delta=\pi(A / K)^{1 / 2}\right.$ where $A$ is the exchange constant and $K$, the MCA constant] with modified exchange and anisotropy constants $A^{\prime}$ and $K^{\prime}$, then $H_{u}$ is given by ${ }^{13}$

$$
\frac{H_{u}}{H_{a}} \simeq \alpha \frac{w}{\delta}\left(\frac{A}{A^{\prime}}-\frac{K^{\prime}}{K}\right),
$$

with $\alpha=\pi / 3^{3 / 2} \simeq 0.60$. However, the "slab" picture does not make sense in hard magnets $(\delta \sim 5-10 \mathrm{~nm})$ since $A$ converges very slowly in real space ${ }^{14}$ and may not be regarded as a local property at the nanometer length scale. Hence the "exchange part" of $H_{u}$ must be found using the microscopic representation of the APB as the maximum field at which an equilibrium state of a domain wall at the APB is possible. The exchange parameters $J_{i j}$ may be calculated using the method of Ref. 15.

The modification of $K$ at the defect may be accounted for perturbatively. Therefore $H_{u}$ may be divided in two parts, $H_{u}=H_{u e}+H_{u a}$, where $H_{u a}=\alpha H_{a}(w / \delta)\left(1-K^{\prime} / K\right)$, and $H_{u e}$ must be found as described above assuming that MCA is not affected by the APB. Assuming that the perturbation of $K$ from the APB falls off at distances smaller than $\delta$, we may write for $H_{u a}$ :

$$
H_{u a}=\alpha H_{a} \Delta f_{a} / K \delta,
$$

where $\Delta f_{a}$ is the "anisotropy deficit" per unit area of the defect, i.e., the difference between the total MCA of a sample without and with the defect. The value $\Delta \epsilon_{a}$ of $\Delta f_{a}$ at $T=0$ may be found using first-principles calculations for a supercell. At low temperatures compared to the ordering transition the atomic structure of defects is almost independent on temperature, and it is reasonable to expect that $\Delta f_{a}$ and $K$ depend on $T$ in a similar way. One should bear in mind that symmetry is lowered at the defect, and MCA modification at the defect may lack the easy-axis symmetry of the pure material.
It was suggested earlier that pinning at APBs may be a source of coercivity in CoPt type magnets. ${ }^{16,17}$ Using the parameters obtained from the tight-binding linear-muffin-tinorbital (TBLMTO) method we estimated $H_{u e}$ for a (101)oriented APB in CoPt, and $H_{u a}$ for the same APB in CoPt, $\mathrm{FePt}$, and $\mathrm{FePd}$. We found that $H_{u e}$ is quite small in CoPt and corresponds to domain wall repulsion from the APB with $\left|H_{u e}\right|<1 \mathrm{kOe}$. On the other hand, the MCA is strongly suppressed at the (101) APB. The values of $\Delta \epsilon_{a}$ per area of the APB corresponding to one formula unit in each atomic layer are $5.0 E_{a}$ for $\mathrm{CoPt}, 2.8 E_{a}$ for $\mathrm{FePt}$, and $4.8 E_{a}$ for $\mathrm{FePd}$, where $E_{a}$ is the bulk MCA energy per formula unit. This MCA suppression leads to a considerable domain wall attraction to APBs. Using Eq. (3) and the values of $H_{a}$ and $\delta$ from Ref. 8 we find ${ }^{18} H_{u e} \simeq 11,7$, and $1.5 \mathrm{kOe}$ for $\mathrm{CoPt}$, FePt, and FePd, respectively. These values notably exceed maximum observed coercivities and indicate that domain wall interaction with APBs may strongly affect the magnetization reversal.

\section{DOMAIN WALL SPLITTING AT TWIN BOUNDARIES}

The structure of domain walls in polytwinned magnets was studied using the microscopic approach. ${ }^{19}$ The magnetic alloy is described by the classical Hamiltonian with magnetic moments localized at the lattice sites. The Hamiltonian includes Heisenberg exchange, dipole-dipole interaction, MCA term, and interaction with external field. The equilibrium states are found by minimizing the free energy in the mean-field approximation.

The equilibrium structure of macrodomain walls (domain walls crossing the twinned stack) in CoPt was found ${ }^{19}$ for $(1 \overline{1} 0)$ and (001) orientations in a stack with perfect (110) twin boundaries. Macrodomain walls of both orientations have a peculiar feature: the domain wall segments in adjacent $c$-domains are displaced with respect to each other. This displacement lowers the exchange energy at domain wall intersections with twin boundaries because magnetization within each domain wall segment becomes parallel to that in the adjacent $c$ domains. The displacement also increases the magnetostatic energy due to the appearance of magnetic charges at the twin boundaries, but due to the small value of the magnetostatic parameter $\eta=2 \pi M^{2} / K(\sim 0.1$ in $\mathrm{CoPt})$ this increase is unimportant at the length scale of $\delta$. Both exchange and dipole energies are lower when the displacements alternate regularly in the stack; in this case the macrodomain wall as a whole is magnetically uncharged and has no long-range magnetic field.

An important property of macrodomain walls is that their segments are coupled only by weak magnetostatic forces $(\eta \ll 1)$. If the latter were absent $(\eta \rightarrow 0$ limit $)$, there would be no interaction between domain wall segments at distances greater than $\delta$. At $\eta \neq 0$ the macrodomain walls may be split in "partial macrodomain walls" by an external field. ${ }^{20}$ For example, consider a stack formed by twinned domains with tetragonal axes pointing along $x$ and $y$ axes of the parent fcc lattice. If the domain wall segments in $y$ domains are held in place (pinned), and those in $x$ domains are not, then an external field above a certain "splitting thresh- 
old" $H_{\mathrm{sp}}$ in the $x$ direction detaches the partial macrodomain wall composed of segments in $x$ domains and moves it away from the pinned partial wall in $y$ domains. It is easy to estimate $H_{\mathrm{sp}}$ micromagnetically. ${ }^{20}$ In particular, for a (001) macrodomain wall in a stack with $d \gg \delta$ ( $d$ being the thickness of twins) $H_{\mathrm{sp}}=\pi M$, and at $d \leqslant \delta$ it is even smaller. In CoPt type magnets $H_{\mathrm{sp}} \sim 3 \mathrm{kOe}$. Obviously, magnetization reversal involves macrodomain wall splitting and further displacement of partial macrodomain walls.

\section{COERCIVITY}

Some general features of the dependence of the coercive force $H_{c}$ on $H_{u}, H_{s}$, and APB density may be deduced without any simulations. ${ }^{20}$ To this end we assume that the APB distribution in each twin may be approximated as an array of similar pinning centers with the typical distance $l_{d}$ between them, and identify $H_{u}$ with the field required to unpin a domain wall segment from any of these centers. We restrict ourselves to the case of external field $\mathbf{H}_{0}$ parallel to the $x$ axis in a single crystal with different types of polytwinned stacks. Such field does not affect $y z$ stacks, except for a reversible transverse magnetization. In other stacks $\mathbf{H}_{0}$ exerts a force only on $x$ segments. If $H_{\mathrm{sp}}<H_{u}$, the macrodomain wall may split in two partial walls at sufficiently large $\mathbf{H}_{0}$. Each domain wall segment may be effectively pinned, and the coercive force $H_{c} \simeq H_{u}+H_{\mathrm{sp}}$. If $H_{\mathrm{sp}}>H_{u}$, the macrodomain wall can only move as a whole. Here we need to introduce an additional parameter $l_{m}$, "the allowed segment displacement," defined as the maximum splitting of a macrodomain wall (displacement between the two partial walls) possible without unpinning the partial wall in $y$ or $z$ domains. It follows that if $l_{m}>l_{d}$, the effective unpinning field of the macrodomain wall is $H_{U} \sim 2 H_{u}$. However, as $l_{m}$ becomes smaller than $l_{d}, H_{U}$ is quickly reduced because only a fraction of domain wall segments may be pinned simultaneously.

\section{REALISTIC ILLUSTRATION}

The complicated interaction of domain walls with twin boundaries and APBs should be studied for realistic microstructures. To provide a starting point for such studies, Fig. 1 shows a stable configuration of two macrodomain walls in the CoPt model with a simulated microstructure ${ }^{21}$ similar to the experimental one shown in Fig. 5 of Ref. 12. This microstructure is similar to that shown in Fig. 5 of Ref. 11, but it was obtained with a larger tetragonal distortion $\epsilon_{m}=0.2$. The simulation box contains $256 \times 256 \times 1$ body-centered tetragonal (bct) cells. The bct representation of the fcc lattice is convenient because it allows us to align the edges of the simulation box perpendicular to the macrodomain walls, thus facilitating the use of periodic boundary conditions. As in the studies of domain wall splitting above, we added the magnetic degrees of freedom to the microscopically defined microstructure and used the microscopic approach ${ }^{19}$ to find the equilibrium configuration of macrodomain walls. For each lattice site the direction of the easy magnetization axis $\mathbf{e}_{i}$ (coinciding with the local direction of the tetragonal axis) and magnitude of MCA energy $b_{i}$ are specified. The APBs

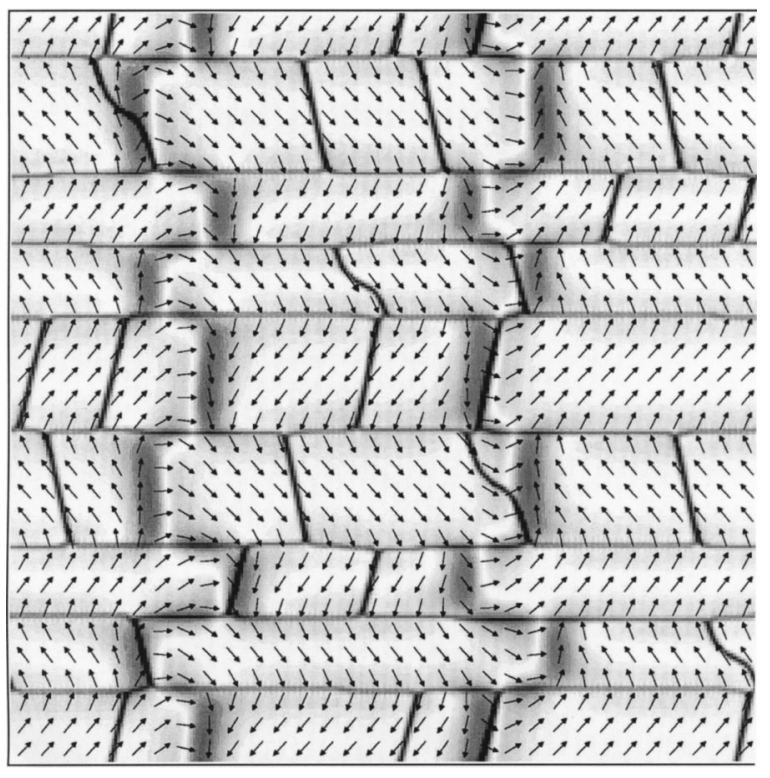

FIG. 1. Macrodomain walls simulated for the model of a polytwinned CoPt magnet (quasi-two-dimensional box with $\sim 70 \mathrm{~nm}$ edge). Arrows show the magnetization; grayscale shows the absolute magnetic charge density, $|\operatorname{div} \mathbf{M}|$. The black lines show horizontal twin boundaries and APBs within the twins.

were identified using a suitable combination of local order parameters, and the parameters $b_{i}$ were set to zero in the close vicinity of APBs so that to reproduce the domain wall attraction to the APBs obtained above for CoPt. The exchange parameters $J_{i j}$ for nearest and next-nearest neighbors were chosen as $J_{2} / J_{1}=2 / 3, J_{3} / J_{1}=1 / 6$; with this choice the spin stiffness is isotropic in the bulk and continuous at (101) APBs. This choice neglects the possible effects associated with spin stiffness anisotropy which will be studied elsewhere, ${ }^{22}$ and also reflects the weak modification of exchange interaction at APBs. This simulation corresponds to the $H_{u}>H_{s}$ case discussed above.

\section{CONCLUSION}

From the methodical point of view, we have shown that coercivity of hard magnets is an inherently multiscale property. Although some aspects of the problem of magnetization reversal may be studied using a single-length-scale approach, the problem as a whole requires a more complicated description. One of the perspectives adopted here is that multiscale modeling should be seen as a way of answering to our inability to rely on pure "brute force" approaches in modeling. We believe that such a synergistic type of approach is a prerequisite for success for any theory in the area of nanoscale magnets.

In this article we presented general guidelines for the consistent multiscale treatment of hysteretic phenomena in hard magnets. We showed how first-principles calculations may be used to obtain crucial information about the shortrange domain wall interaction with defects required for micromagnetic simulations. Short-range properties are studied using microscopic Heisenberg-model calculations with $a b$ initio parameters. These properties characterize the defects viewed as geometrical objects and may be parametrized in 
any convenient way at the micromagnetic length scales exceeding the domain wall width $\delta$. Together with the simulations of microstructural evolution these techniques are combined into a powerful, completely theoretical approach to hysteretic phenomena.

While the multiscale framework of this approach is more or less universal, the appropriate tools for its realization are material-specific. For rare-earth-based magnets some of these tools are still lacking. However, for such magnets as CoPt the first-principles calculations are practical enough to make semiquantitative predictions, while microstructural evolution is also well described theoretically. Application of these techniques within the framework of our synergistic approach revealed $^{18}$ that coercivity of CoPt type magnets is a complex multiscale property emerging as a combined effect of domain wall pinning at antiphase boundaries and splitting at twin boundaries. The first source develops at the atomistic scale and is determined by the suppression of magnetic anisotropy at antiphase boundaries; the second one due to magnetostatic interaction develops at the characteristic microstructural length scale (distance between the twin boundaries). The importance of multiple length scales for the development of coercivity seems to be a generic feature of all nanoscale magnetic materials.

\section{ACKNOWLEDGMENTS}

This work was carried out at Ames Laboratory, which is operated for the U.S. Department of Energy by Iowa State University under Contract No. W-7405-82. This work was supported by the Director for Energy Research, Office of Basic Energy Sciences of the U.S. Department of Energy.
${ }^{1}$ M. E. McHenry and D. E. Laughlin, Acta Mater. 48, 223 (2000).

${ }^{2}$ J. Schwitalla, B. L. Gyorffy, and L. Szunyogh, Phys. Rev. B 63, 104423 (2001).

${ }^{3}$ T. A. Arias, Rev. Mod. Phys. 71, 267 (1999).

${ }^{4}$ R. E. Rudd and J. Q. Broughton, Phys. Rev. B 58, R5893 (1998); V. V. Dobrovitskii, M. I. Katsnelson, and B. N. Harmon, J. Magn. Magn. Mater. 221, L235 (2000).

${ }^{5}$ H. Kronmüller, R. Fischer, M. Seeger, and A. Zern, J. Phys. D 29, 2274 (1996).

${ }^{6}$ J. Fidler and T. Schrefl, J. Phys. D 33, R135 (2000).

${ }^{7}$ V. P. Antropov, cond-mat/0211144 (2002).

${ }^{8}$ C. Yanar, J. M. K. Wiezorek, and W. A. Soffa, in Phase Transformations and Evolution in Materials, edited by P. E. A. Turchi and A. Gonis (TMS, Warrendale, PA, 2000), p. 39.

${ }^{9}$ N. I. Vlasova, G. S. Kandaurova, and N. N. Shchegoleva, J. Magn. Magn. Mater. 222, 138 (2000).

${ }^{10}$ A. G. Khachaturyan, Theory of Structural Phase Transformations in Solids (Wiley, New York, 1983).

${ }^{11}$ K. D. Belashchenko, I. R. Pankratov, G. D. Samolyuk, and V. G. Vaks, J. Phys.: Condens. Matter 14, 565 (2002).

${ }^{12}$ C. Leroux, A. Loiseau, D. Broddin, and G. Van Tendeloo, Philos. Mag. B 64, 58 (1991).

${ }^{13}$ H. Kronmüller, J. Magn. Magn. Mater. 7, 341 (1978).

${ }^{14}$ V. P. Antropov, B. N. Harmon, and A. N. Smirnov, J. Magn. Magn. Mater. 200, 148 (1999).

${ }^{15}$ M. van Schilfgaarde and V. P. Antropov, J. Appl. Phys. 85, 4827 (1999).

${ }^{16}$ Ya. S. Shur, L. M. Magat, G. V. Ivanova, A. I. Mitsek, A. S. Yermolenko, and O. A. Ivanov, Phys. Met. Metallogr. 26, 241 (1968).

${ }^{17}$ B. Zhang and W. A. Soffa, Scr. Metall. Mater. 30, 683 (1994).

${ }^{18}$ K. D. Belashchenko and V. P. Antropov, J. Magn. Magn. Mater. 253, L87 (2002).

${ }^{19}$ K. D. Belashchenko and V. P. Antropov, J. Appl. Phys. 91, 8474 (2002).

${ }^{20}$ K. D. Belashchenko and V. P. Antropov, Phys. Rev. B 66, 144402 (2002).

${ }^{21}$ I. R. Pankratov and V. G. Vaks (unpublished).

${ }^{22}$ K. D. Belashchenko (unpublished). 